\title{
PET/CT imaging for evaluating response to therapy in castration-resistant prostate cancer
}

\author{
Francesco Ceci $^{1,2} \cdot$ Paolo Castellucci $^{1} \cdot$ Cristina Nanni $^{1} \cdot$ Stefano Fanti $^{1}$
}

Published online: 25 August 2016

(C) Springer-Verlag Berlin Heidelberg 2016

Prostate cancer $(\mathrm{PCa})$ represents the most common malignancy in men [1], and begins as an androgen-dependent tumour. Androgen deprivation therapy (ADT) represents the backbone of treatment for relapsed or advanced prostate cancer patients. However, after a median of 12-24 months of initial response, disease progression usually occurs, despite castrate levels of serum testosterone ( $\leq 50 \mathrm{ng} / \mathrm{dL})$, defining a state called castration-resistant prostate cancer (CRPC) [2]. Metastatic CRPC (mCRPC) in advanced disease is associated with high mortality rates (overall survival [OS] of 2-3 years) [1]. In these patients, palliation is the treatment of choice, and many different therapies are available nowadays, including taxanebased chemotherapy and novel androgen receptor (AR)targeted therapy [3]. However, a significant number of patients develop resistance, showing no response or showing progression during therapy. Thus, the early identification of non-responders is crucial for avoiding the administration of toxic, futile and expensive therapies.

The assessment of PSA levels over time is routinely used both to evaluate response to therapy and to predict outcomes $[1,3]$. PSA levels have been shown to correlate with disease burden [4] and are included in many prognostic tools for estimating survival. This correlation becomes more complicated

This Editorial Commentary refers to the article http://dx.doi.org/10.1007 /s00259-016-3439-9

Francesco Ceci

francesco.ceci@studio.unibo.it

1 Nuclear Medicine, S.Orsola-Malpighi University Hospital, University of Bologna, Bologna, Italy

2 UO Medicina Nucleare PAD. 30, Azienda Ospedaliero-Universitaria di Bologna, Policlinico S.Orsola-Malpighi, Via Massarenti, 9, 40138 Bologna, Italy in castrate disease states [5], which are characterized by increased disease heterogeneity due to the complex mechanisms surrounding CRPC development [6]. Thus, PSA is not a reliable marker [3], since it can be impaired by flair phenomena [7], tumour cell heterogeneity [6], and active visceral metastases not producing PSA [8]. Increasing PSA levels predict disease progression with good accuracy; however, decreasing PSA is not always related to homogeneous response to therapy [9].

Conventional imaging techniques with computed tomography (CT), magnetic resonance (MRI) and bone scintigraphy (BS) are late indicators of treatment efficacy [1,3]. The evaluation of changes provided by PET/CT rather than morphological changes during therapy may be an early and reliable alternative to other indicators of treatment benefit such as radiological progression-free survival (rPFS) and PSA. Thus, PET/CT functional imaging, which enables an evaluation of metabolism or receptor expression, has proved to be a valid tool for early prediction of response to therapy in many solid tumours.

There are already a few preliminary reports in the literature supporting the potential of choline PET/CT as an indicator of therapy efficacy in mCRPC patients. De Giorgi et al. [10, 11] reported on two studies in which they investigated the role of 18F-choline PET/CT for evaluating early response to treatment with abiraterone and enzalutamide, respectively. The response assessed with choline PET/CT was associated with more favourable OS than a reduction in PSA of $\geq 50 \%$. Thus, the combination of PET/CT and PSA could be a valid tool for early prediction of outcome and therapy monitoring. Maines et al. [12] confirmed these data in $30 \mathrm{mCRPC}$ patients treated with enzalutamide. They observed that maximum standardized uptake values (SUVmax) measured at pre-enzalutamide PET were significantly associated with rPFS and OS. Ceci et al. [9] studied a cohort of $61 \mathrm{mCRPC}$ patients in which 
11C-choline-PET/CT was performed before and after treatment with docetaxel. Interestingly, 11C-choline-PET/CT showed disease progression in the $44 \%$ of patients who showed a reduction in PSA of $\geq 50 \%$ after docetaxel. The tumor burden, expressed as more than ten PET-positive bone lesions measured before docetaxel, was also significantly associated with an increased probability of progression after treatment.

The latest study on this topic published in the European Journal of Nuclear Medicine and Molecular Imaging was conducted by Schwarzenböck et al. [13]. The authors prospectively evaluated the value of $11 \mathrm{C}$-choline PET/CT in monitoring early and late response to a standardized first-line treatment with docetaxel in a cohort of $32 \mathrm{mCRPC}$ patients. Patients were referred for 11C-choline PET/CT before docetaxel and after one and ten chemotherapy cycles. The results of PET/CT were compared to the RECIST 1.1 and clinical criteria-based therapy response assessment, including PSA, for defining progressive disease (PD) and non-PD, respectively. No significant correlation was observed between changes in choline uptake in 11C-choline PET/CT and therapy response assessment based on RECIST 1.1 and clinical criteria during the early and late course of docetaxel. As a consequence, the authors suggested limited use of choline PET/ $\mathrm{CT}$ for assessing response to therapy in standardized firstline docetaxel. In our opinion, and as correctly reported by the authors, the study presents some limitations: 1) the use of RECIST criteria as a parameter for defining response to treatment, especially in the evaluation of bone metastases (increased sclerosis of bone metastases during therapy might be misinterpreted as PD);2) the limited number of patients enrolled; and 3) the assessment of response to therapy based on analysis of patients at a group rather than individual level.

Beyond choline, new tracers have been proposed for investigating PCa. Anti-3-18F-FACBC and 68Ga-HBED-CCPSMA have shown the most promising results. These radiotracers demonstrated higher sensitivity and specificity than choline for restaging PCa. Nevertheless, the efficacy of these procedures as indicators of treatment efficacy and as predictors of patient outcome in mCRPC patients has not yet been assessed.

The availability of a procedure such as PET/CT (with choline or other radiotracers) for accurate and early assessment of response to chemotherapy and AR-targeted therapy will have an important impact on the management of mCRPC. It could certainly lead to more tailored therapy, especially for patients presenting reduced PSA levels during treatment while imaging may show progression. In fact, these patients could be switched to a second line of systemic therapy, to radiotherapy on the non-responding lesions, or to radium-223. Considering the relevant cost of these therapies, the availability of a diagnostic test able to predict treatment response (earlier than laboratory and conventional imaging) could represent a promising approach for monitoring $\mathrm{mCRPC}$ and saving time and money. As a consequence, in addition to improved life expectancy, the collateral effects/toxicity and costs of futile therapy would be reduced.

\section{Compliance with ethical standards}

Conflict of interest The authors declare that they do not have a conflict of interest.

\section{References}

1. Heidenreich A, Bastian PJ, Bellmunt J, Bolla M, Joniau S, van der Kwast T, et al. EAU guidelines on prostate cancer. Part II: treatment of advanced, relapsing, and castration-resistant prostate cancer. Eur Urol. 2014;65:467-79.

2. Chen CD, Welsbie DS, Tran C, et al. Molecular determinants of resistance to antiandrogen therapy. Nat Med. 2004;10(1):33-9.

3. Scher HI, Morris MJ, Stadler WM, Higano C, Basch E, Fizazi KS, et al. Trial design and objectives for castration-resistant prostate cancer: updated recommendations from the Prostate Cancer Clinical Trials Working Group 3. J Clin Oncol. 2016;34(12): 1402-18.

4. Carvalhal GF, Daudi SN, Kan D, Mondo D, Roehl KA, Loeb S, et al. Correlation between serum prostate-specific antigen and cancer volume in prostate glands of different sizes. Urology. 2010;76: 1072-6.

5. Halabi S, Lin CY, Kelly WK, Fizazi KS, Moul JW, Kaplan EB, et al. Updated prognostic model for predicting overall survival in first-line chemotherapy for patients with metastatic castrationresistant prostate cancer. J Clin Oncol. 2014;32:671-7.

6. Robinson D, Van Allen EM, Wu YM, Schultz N, Lonigro RJ, Mosquera JM, et al. Integrative clinical genomics of advanced prostate cancer. Cell. 2015;161:1215-28.

7. Burgio SL et al. PSA flare with abiraterone in patients with metastatic castration-resistant prostate cancer. Clin Genitourin Cancer. 2015;13(1):39-43.

8. Pezaro CJ, Omlin A, Lorente D, Nava Rodrigues D, Ferraldeschi R, Bianchini D, et al. Visceral disease in castration-resistant prostate cancer. Eur Urol. 2014;65:270-3.

9. Ceci F, Castellucci P, Graziani T, Schiavina R, Renzi R, Di Tullio P, et al. 11C-choline PET/CT in castration-resistant prostate cancer patients treated with docetaxel. Eur J Nucl Med Mol Imaging. 2016;43(1):84-91.

10. De Giorgi U, Caroli P, Burgio SL, Menna C, Conteduca V, Bianchi E, et al. Early outcome prediction on 18F-fluorocholine PET/CT in metastatic castration-resistant prostate cancer patients treated with abiraterone. Oncotarget. 2014;5:12448-58.

11. De Giorgi U, Caroli P, Scarpi E, Conteduca V, Burgio SL, Menna C, et al. (18) F- Fluorocholine PET/CT for early response assessment in patients with metastatic castration- resistant prostate cancer treated with enzalutamide. Eur J Nucl Med Mol Imaging. 2015;42: 1276-83.

12. Maines F, Caffo O, Donner D, et al. Serial (18)F-Choline-PET imaging in patients receiving enzalutamide for metastatic castration-resistant prostate cancer: response assessment and imaging biomarkers. Future Oncol. 2016;12(3):333-42.

13. Schwarzenböck SM, Eiber M, Kundt G, Retz M, Sakretz M, Kurth J, et al. Eur J NuclMed Mol Imaging. 2016. doi:10.1007/s00259016-3439-9. 